UCRHEP-T410

May 2006

\title{
Common Origin of Neutrino Mass, Dark Matter, and Baryogenesis
}

\author{
Ernest Ma \\ Physics Department, University of California, Riverside, California 92521
}

\begin{abstract}
Combining one established idea with two recent ones, it is pointed out for the first time that three of the outstanding problems of particle physics and cosmology, i.e. neutrino mass, dark matter, and baryogenesis, may have a simple common solution, arising from the interactions of a single term, with experimentally verifiable consequences.
\end{abstract}


The minimal standard model (SM) of particle interactions is very successful, but it fails to address three of the most important qusetions facing particle physics and cosmology today: (1) How is a neutrino mass generated? (2) What is the nature of cold dark matter?

Why is there more matter than antimatter in the present Universe? Each question has several different good answers, but they are generally unrelated. In this note, combining one established idea and two recent ones, it is pointed out for the first time that all three may have a simple common origin, arising from the interactions of a single term, with experimentally verifiable consequences.

It has been known for a long time that neutrino mass and baryogenesis may be related through the canonical seesaw mechanism [1, 2, 3] and leptogenesis [4. The idea is very simple. The SM is augmented by three heavy neutral singlet fermions $N_{i}$ (often referred to as right-handed neutrinos) which have large Majorana masses $M_{i}$. Through the Yukawa couplings

$$
\mathcal{L}_{Y}=h_{\alpha i}\left(\nu_{\alpha} \phi^{0}-l_{\alpha} \phi^{+}\right) N_{i}+H . c .
$$

where $\left(\nu_{\alpha}, l_{\alpha}\right), \alpha=e, \mu, \tau$, are the three left-handed lepton doublets, and $\left(\phi^{+}, \phi^{0}\right)$ is the SM Higgs scalar doublet, the unique dimension-five operator for Majorana neutrino mass [5]

$$
\mathcal{L}_{\Lambda}=\frac{f_{\alpha \beta}}{2 \Lambda}\left(\nu_{\alpha} \phi^{0}-l_{\alpha} \phi^{+}\right)\left(\nu_{\beta} \phi^{0}-l_{\beta} \phi^{+}\right)+H . c .
$$

is realized with $f_{\alpha \beta} / \Lambda=\sum_{i} h_{\alpha i} h_{\beta i} / M_{i}$. As $\phi^{0}$ acquires a nonzero vacuum expectation value $\left\langle\phi^{0}\right\rangle=v$, a neutrino mass matrix $\left(\mathcal{M}_{\nu}\right)_{\alpha \beta}=f_{\alpha \beta} v^{2} / \Lambda$ is generated [1, 2, 3]. At the same time, the heavy Majorana fermions $N_{i}$ may decay into either $\nu \phi^{0}$ and $l^{-} \phi^{+}$or their antiparticles in the early Universe, and establish a lepton asymmetry [4, which gets converted into the present observed baryon asymmetry of the Universe through sphalerons at the electroweak phase transition [6]. The interactions of Eq. (1) together with $M_{i}$ are thus responsible for both neutrino mass and baryogenesis. What is missing is just dark matter. 
To include dark matter, it has recently been recognized [7] that a very simple change of Eq. (1) is all one needs, i.e.

$$
\mathcal{L}_{Y}=h_{\alpha i}\left(\nu_{\alpha} \eta^{0}-l_{\alpha} \eta^{+}\right) N_{i}+H . c .,
$$

where $\left(\eta^{+}, \eta^{0}\right)$ is a second scalar doublet, and an exactly conserved $Z_{2}$ symmetry has been assumed, under which $\left(\eta^{+}, \eta^{0}\right)$ and $N_{i}$ are odd, with all other particles even. This means that $\left\langle\eta^{0}\right\rangle=0$ and $N_{i}$ are $\underline{\text { not }}$ the Dirac mass partners of $\nu_{\alpha}$ as in the canonical seesaw model. Nevertheless, a Majorana neutrino mass matrix $\mathcal{M}_{\nu}$ is obtained in one loop [8], as depicted in FIG. 1.

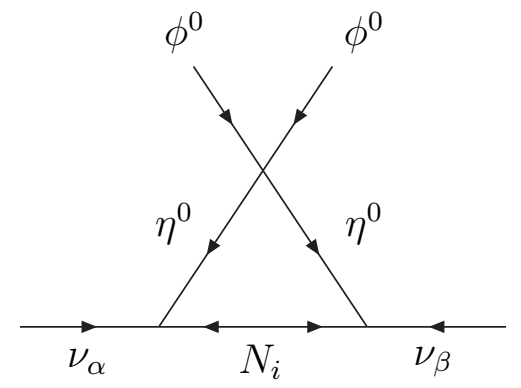

Figure 1: One-loop generation of neutrino mass.

Let $\eta^{0}=\left(\eta_{R}^{0}+i \eta_{I}^{0}\right) / \sqrt{2}$ and $m_{R, I}$ be the mass of $\eta_{R, I}^{0}$, then

$$
\left(\mathcal{M}_{\nu}\right)_{\alpha \beta}=\sum_{i} \frac{h_{\alpha i} h_{\beta i} M_{i}}{16 \pi^{2}}\left[\frac{m_{R}^{2}}{m_{R}^{2}-M_{i}^{2}} \ln \frac{m_{R}^{2}}{M_{i}^{2}}-\frac{m_{I}^{2}}{m_{I}^{2}-M_{i}^{2}} \ln \frac{m_{I}^{2}}{M_{i}^{2}}\right] .
$$

The immediate consequence of the exact $Z_{2}$ symmetry of this model is that the lightest $N_{i}$ or $\eta_{R, I}^{0}$ is a candidate for the cold dark matter of the Universe. Assuming that $N_{i}$ is the cold dark matter, it has been shown [9] that its relic abundance is compatible with observation only for $m_{R, I}$ less than about $350 \mathrm{GeV}$, for which the experimental upper bound of $1.2 \times 10^{-11}$ on the branching fraction of $\mu \rightarrow e \gamma$ implies a lower bound on the mass of the lightest neutrino of order $0.05 \mathrm{eV}$. This result is of course very interesting, but it goes against 
leptogenesis because the interactions of Eq. (3) must then be lepton-number violating at the electroweak scale and would wash out any lepton asymmetry even if it were somehow created at higher energies.

There is however another option. Instead of $N_{i}$, consider $\eta_{R}^{0}$ or $\eta_{I}^{0}$ as the cold dark matter [10. In that case, its relic abundance does not have to depend on the interactions of Eq. (3), but rather on its gauge and scalar interactions. This allows $M_{i}$ to be much heavier than $m_{R, I}$ in Eq. (4) which reduces to

$$
\left(\mathcal{M}_{\nu}\right)_{\alpha \beta}=\sum_{i} \frac{h_{\alpha i} h_{\beta i}}{16 \pi^{2} M_{i}}\left[m_{R}^{2} \ln \frac{M_{i}^{2}}{m_{R}^{2}}-m_{I}^{2} \ln \frac{M_{i}^{2}}{m_{I}^{2}}\right] .
$$

Compared to the canonical seesaw formula of $\left(\mathcal{M}_{\nu}\right)_{\alpha \beta}=\sum_{i} h_{\alpha i} h_{\beta i} v^{2} / M_{i}$, it is reduced by a factor of about $16 \pi^{2}$, assuming $M_{R}^{2} \ln \left(M_{i}^{2} / M_{R}^{2}\right)-M_{I}^{2} \ln \left(M_{i}^{2} / M_{I}^{2}\right)$ is of order $v^{2}$. This means that the Davidson-Ibarra bound [11] on leptogenesis is now reduced by $16 \pi^{2}$, and the lightest $N_{i}$ needs only to be heavier than about $2.5 \times 10^{7} \mathrm{GeV}$, well below a possible gravitino bound of $10^{9} \mathrm{GeV}$. In the decay of $N_{i}$ to either $\nu \eta^{0}$ and $l^{-} \eta^{+}$or their antiparticles, not only is a lepton asymmetry created, but also dark matter. At temperatures below the smallest $M_{i}$, of order $10^{7}$ to $10^{11} \mathrm{GeV}$, the interactions of Eq. (3) are frozen out and the lepton asymmetry remains and will be converted to the observed baryon asymmetry of the Universe through sphalerons in the usual way [12. At the same time, because of the $\lambda_{5}$ term to be discussed later, there is no corresponding dark-matter asymmetry, and the annihilation of $\eta^{0}$ at a temperature when it becomes nonrelativistic will determine its relic abundance [13, as shown in Ref. [10]. The stated goal of having a simple common origin, i.e. Eq. (3), for neutrino mass, dark matter, and baryogenesis has been realized.

The next question to consider is this model's experimental consequences. At the electroweak scale, its particle content is identical to that of Ref. [10], except that neutrinos have mass here according to Eq. (5), but not there. The scalar sector consists of two doublets: $\left(\phi^{+}, \phi^{0}\right)$ as in the SM, and the new all-important $\left(\eta^{+}, \eta^{0}\right)$. Their scalar interactions are given 
by

$$
\begin{aligned}
V & =m_{1}^{2} \Phi^{\dagger} \Phi+m_{2}^{2} \eta^{\dagger} \eta+\frac{1}{2} \lambda_{1}\left(\Phi^{\dagger} \Phi\right)^{2}+\frac{1}{2} \lambda_{2}\left(\eta^{\dagger} \eta\right)^{2}+\lambda_{3}\left(\Phi^{\dagger} \Phi\right)\left(\eta^{\dagger} \eta\right) \\
& +\lambda_{4}\left(\Phi^{\dagger} \eta\right)\left(\eta^{\dagger} \Phi\right)+\frac{1}{2} \lambda_{5}\left[\left(\Phi^{\dagger} \eta\right)^{2}+H . c .\right]
\end{aligned}
$$

where $\lambda_{5}$ has been chosen real without any loss of generality. For $m_{1}^{2}<0$ and $m_{2}^{2}>0$, only $\phi^{0}$ acquires a nonzero vacuum expectation value $v$. The masses of the resulting physical scalar bosons are given by

$$
\begin{aligned}
m^{2}\left(\phi_{R}^{0}\right) & =2 \lambda_{1} v^{2} \\
m^{2}\left(\eta^{ \pm}\right) & =m_{2}^{2}+\lambda_{3} v^{2} \\
m^{2}\left(\eta_{R}^{0}\right) & =m_{2}^{2}+\left(\lambda_{3}+\lambda_{4}+\lambda_{5}\right) v^{2} \\
m^{2}\left(\eta_{I}^{0}\right) & =m_{2}^{2}+\left(\lambda_{3}+\lambda_{4}-\lambda_{5}\right) v^{2}
\end{aligned}
$$

Since $\eta^{ \pm}$cannot be dark matter, $\lambda_{4} \pm \lambda_{5}<0$ is required. If $\lambda_{5}<0$ is also chosen, then $\eta_{R}^{0}$ is lighter than $\eta_{I}^{0}$, and becomes the dark-matter candidate of this model.

The $\eta$ particles can be produced in pairs directly by the SM gauge bosons $W^{ \pm}, Z$, or $\gamma$. Once produced, $\eta^{ \pm}$will decay into $\eta_{R, I}^{0}$ and a virtual $W^{ \pm}$, which becomes a quark-antiquark or lepton-antilepton pair, and $\eta_{I}^{0}$ will decay into $\eta_{R}^{0}$ and a virtual $Z^{0}$. The decay chain

$$
\eta^{+} \rightarrow \eta_{I}^{0} l^{+} \nu, \quad \text { then } \eta_{I}^{0} \rightarrow \eta_{R}^{0} l^{+} l^{-}
$$

has 3 charged leptons and large missing energy, and can be compared to the direct decay

$$
\eta^{+} \rightarrow \eta_{R}^{0} l^{+} \nu
$$

to extract the masses of the respective particles.

The direct detection of $\eta_{R}^{0}$ in present dark-matter search experiments is discussed in Ref. [10, where it is found to be about two orders of magnitude below current experimental 
capabilities. The additional contribution from Eq. (3) is negligible because $\eta_{R}^{0}$ interacts directly only with neutrinos, and $N_{i}$ are assumed very heavy. Contribution to the one-loop radiative process $\mu \rightarrow e \gamma$ is also negligible, in contrast to the case where $N_{i}$ play the role of dark matter [9].

To conclude, it has been pointed out in this note that neutrino mass, dark matter, and baryogenesis may have a simple common origin, i.e. Eq. (3). The proposed model [7] uses the result of Ref. [10 that $\eta_{R}^{0}$ is a suitable bosonic dark-matter candidate, and that of canonical leptogenesis [4]. It predicts rather small cross sections for $\eta_{R}^{0}$ detection at low energies, although future experiments will become sensitive to it. The decays of $\eta^{ \pm}$and $\eta_{I}^{0}$ into $\eta_{R}^{0}$ should be observable at the forthcoming Large Hadron Collider (LHC).

I thank M. Frigerio, T. Hambye, and J. Kubo for useful discussions. This work was supported in part by the U. S. Department of Energy under Grant No. DE-FG03-94ER40837. 


\section{References}

[1] T. Yanagida, in Proceedings of the Workshop on the Unified Theory and the Baryon Number in the Universe, edited by O. Sawada and A. Sugamoto (KEK Report No. 7918, Tsukuba, Japan, 1979), p. 95.

[2] M. Gell-Mann, P. Ramond, and R. Slansky, in Supergravity, edited by P. van Nieuwenhuizen and D. Z. Freedman (North-Holland, Amsterdam, 1979), p. 315.

[3] R. N. Mohapatra and G. Senjanovic, Phys. Rev. Lett. 44, 912 (1980).

[4] M. Fukugita and T. Yanagida, Phys. Lett. B174, 45 (1986).

[5] S. Weinberg, Phys. Rev. Lett. 43, 1566 (1979).

[6] V. A. Kuzmin, V. A. Rubakov, and M. E. Shaposhnikov, Phys. Lett. 155B, 36 (1985).

[7] E. Ma, Phys. Rev. D73, 077301 (2006).

[8] E. Ma, Phys. Rev. Lett. 811171 (1998).

[9] J. Kubo, E. Ma, and D. Suematsu, hep-ph/0604114.

[10] R. Barbieri, L. J. Hall, and V. S. Rychkov, hep-ph/0603188.

[11] S. Davidson and A. Ibarra, Phys. Lett. B535, 25 (2002).

[12] For a recent review, see for example W. Buchmuller, R. D. Peccei, and T. Yanagida, Ann. Rev. Nucl. Part. Sci. 55, 311 (2005).

[13] For a recent review, see for example G. Bertone, D. Hooper, and J. Silk, Phys. Rept. 405, 279 (2005). 